Revista Latinoamericana de la Papa. 24 (2): 42 - 53, 2020

Recibido: 27/07/2020 | Aceptado: 15/10/2020

Publicado en línea: Diciembre 2020

DOI: 10.37066/ralap.v24i2.407

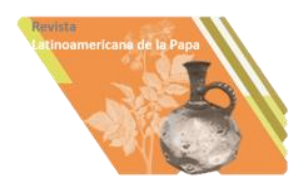

ISSN: 1853-4961

\title{
Nueva variedad de papa ÑUST'A-CICA resistente a Phytophthora infestans (Mont. de Bary)
}

\section{Teofilo Pompeyo Cosio Cuentas ${ }^{1}$; Wilfredo Catalán Bazán ${ }^{2 *}$; Edit Chilo Yepez $^{3}$}

Resumen

El Centro de Investigación en Cultivos Andinos (CICA) de la Universidad Nacional San Antonio Abad del Cusco (UNSAAC) tiene como objetivo el mejoramiento genético de los cultivares nativos. La investigación tiene el objetivo específico de mejoramiento de papa para resistencia a enfermedades, rendimiento de tubérculo y calidad culinaria. El CICA libero una nueva variedad de papa NUST'A-CICA, como una alternativa tecnológica, con características agronómicas superiores para uso de los agricultores de la región y el país. La UNSAAC con fondos CANON entre 2011 a 2015, subvencionó el Proyecto de investigación de Conservación ex situ de Germoplasma de Papa y Liberación de Variedades, que permitió financiar la última fase de esta investigación. La variedad NUUST`A-CICA, después de 16 años de evaluación demostró estabilidad productiva con un rendimiento de tubérculo promedio superior a $40 \mathrm{t} / \mathrm{ha}^{-1}$, calidad de tubérculo de carne amarilla, con $26 \%$ de materia seca y alta resistencia a Phytophthora infestans (Mont. de Bary). Se observa baja incidencia de Sclerotinia, de PVX, PVY y PLRV. Las evaluaciones permitieron afirmar que esta variedad muestra poca preferencia de los insectos Premnotrypes Sp., Tequus spp., Atomopteryx spp; mientras en almacén a la polilla de la papa: Phthorimaea operculella y Synmetrischema tangolias, por sus bajos porcentajes de daño. No se tiene información definitiva sobre resistencia a nematodos, observándose daño esporádico de Globodera spp., en las raíces. Una característica de marcador genético de ÑUST'A-CICA es su autoincompatibilidad, teniendo polen viable y estigmas receptivos, probados en otras cruzas.

Palabras clave: Variedad, nativas, tetraploide, rendimiento, enfermedades.

* Autor para correspondencia. E-mail: wilfredo.catalan@unsaac.edu.pe

1 Dr. Teofilo Pompeyo Cosio Cuentas. (D) Obtentor Vegetal, Docente investigador del Centro de Investigación en Cultivos Andinos. FAZ-UNSAAC.

2 Mg. Sc. Wilfredo Catalán Bazán. (D) Agronomista, Docente investigador del Centro de Investigación en Cultivos Andinos. FAZ-UNSAAC.

3 Ing. Agr. Edit Chilo Yepez. DAgronomista, Asistente investigadora del Programa de Papa del Centro de Investigación en Cultivos Andinos. FAZ-UNSAAC. 


\section{New variety of potato "NUST'A-CICA" resistant to Phytophthora infestans (Mont. de Bary)}

\section{Summary}

The Research Center of Andean Crops Andean (CICA), of the National University of San Antonio Abad of Cusco (UNSAAC), Its primary objective in its potato program is the genetic improvement of native potato cultivars. This research work corresponds to the specific breeding objectives for disease resistance, tuber yield and culinary quality. CICA delivered the new variety of potato " $\tilde{N} U S T{ }^{\prime} A-C I C A$ ", as a new technological alternative, with superior agronomic characteristics for the use of farmers in the region and the country. The UNSAAC through the Vice-Rectorate for Research between 2011 and 2015; subsidized the Ex situ Conservation Research Project of Potato Germplasm and Variety Liberation, which made it possible to finance the last phase of this research. The variety NUST'A-CICA, after 16 years of evaluation, showed a productive stability with an average tuber yield of more than $40 t \cdot \mathrm{ha}^{-1}$, quality of tuber, yellow meat with $26 \%$ of dry matter and high resistance to Phytophthora infestans (Mont de Bary). A low incidence of Sclerotinia is observed, also of PVX, PVY and PLRV.

The long period of evaluation allows to affirm that this variety shows the non-preference of the insects Premnotrypes spp., Tequus spp., Atomopteryx spp., and in storage for the potato moth, Phthorimaea operculella and Synmestrichema tangolias, showed low percentages of damage. There is no definitive information on nematode resistance, with sporadic damage to Globodera spp. in the roots. A genetic marker characteristic of NUST'A-CICA is its self-incompatibility, having viable pollen and receptive stigmas, proven in other crosses.

keywords: Variety, native, tetraploid, yield, diseases

\section{Introducción}

Después de 16 años de investigación el Centro de Investigación en Cultivos Andinos (CICA) de la Universidad Nacional San Antonio Abad del Cusco (UNSAAC) con sede en el Centro Agronómico K'ayra, liberó una nueva alternativa tecnológica de la variedad de papa NUST A-CICA, para la producción de los agricultores, asociaciones de semilleristas e instituciones del sector agrario para que a su vez promuevan su distribución en la región y el país.

La UNSAAC mediante el Vicerrectorado de Investigación entre 2011 a 2015, subvencionó el Proyecto de investigación de Conservación ex situ de Germoplasma de Papa y Liberación de Variedades, que permitió la liberación de esta nueva variedad de papa.
Esta variedad tiene como progenitores fundamentalmente papas nativas de alta resistencia a enfermedades y plagas de la Región y especialmente a Phytophthotra infestans, lo que ha permitido obtener un clon de caracteres agronómicos superiores como lo es KI-SC-29B que constituye la nueva variedad NUST'ACICA.

Durante el largo período de investigación, participaron estudiantes de la Escuela Profesional de Agronomía, en las fases de caracterización y evaluación, realizando tesis para Ingeniero Agrónomo. También participaron agricultores de comunidades campesinas mediante la metodología de investigación participativa en su proceso de validación.

Esta nueva variedad no desplaza la variabilidad de papa nativa, por cuanto 
entre miles de variedades nativas, los nuevos recombinantes con mayor productividad y mejores capacidades de resistencia a factores adversos, se incorporan a los sistemas productivos sin desplazar a las nativas, pero le otorga al agricultor mayores posibilidades de seguridad de cosecha y por lo tanto contribuye a la seguridad alimentaria, bajo las condiciones actuales del cambio climático.

En este contexto, la nueva variedad de papa NUUST'A-CICA, es fundamentalmente un genotipo andígena. Los progenitores "Yana Maqt'illo" y "Sayno Conejo (mostraron alta disposición) en la transferencia de sus caracteres genéticos y agrupan genes de alta productividad y resistencias, hecho que se manifiesta en sus progenies en las cruzas específicas realizadas.

Los objetivos de este trabajo de investigación corresponden al primer objetivo del Programa de papa del CICA: Realizar el mejoramiento genético de las papas nativas de la Región de Cusco. Sus objetivos específicos son mejorar la resistencia a Phytophthora infestans, rendimiento de tubérculo y la calidad del tubérculo.

\section{Materiales y métodos}

El presente trabajo se realizó en el Centro agronómico K'ayra, a 3300 Msnm, la ubicación de los lugares de evaluación y las altitudes correspondientes se expone en la tabla 1.

Los fitomejoradores que obtuvieron la variedad de papa NUST A-CICA, son docentes de Agronomía de la UNSAAC, con amplia experiencia en la conservación de papas nativas in situ y haber asesorado más de 10 tesis para ingeniero agrónomo sobre caracterización y evaluación de más de 1000 cultivares nativos de la región. Las tesis fueron realizadas en diferentes comunidades donde los agricultores mantienen la variabilidad en sus parcelas. El CICA, en este momento mantiene una colección superior de 120 variedades nativas (Core collection) con fines de mejoramiento. No se dispone de un Banco de germoplasma con fines de conservación. En este proceso se identificó cultivares nativos con alta capacidad productiva y resistencias, con los que se planificó en 1995 un conjunto de cruzamientos, de cuyos segregantes se seleccionó esta nueva variedad.

\section{Progenitores de la nueva variedad NUUSTA-CICA.}

Los progenitores que intervinieron en la generación de la nueva variedad ÑUST'A-CICA, fueron:

MICAELA BASTIDAS, Hibrido mejorado (Ochoa, 2003). Obtenido de la cruza de Solanum tuberosum subsp., tuberosum X Solanum tuberosum subsp., adigenum (Ochoa, 2003).Variedad poco conocida por su distribución limitada en la Región de Cusco, se reconoce sus caracteres favorables de precocidad, tallo grueso, estolones cortos y alta calidad del tubérculo. Una característica muy importante es su androesterilidad e hipersensibilidad a los virus PVX y PVY y otra característica muy distinguible es la tonalidad verde amarillenta (virescente) de toda la planta (Cosio, 2005)

YANA MAQT'ILLO, Solanum tuberosum subsp. andigena (Juz. et Buk.) Hawkes $(2 \mathrm{n}=4 \mathrm{x}=48)$ (Hawkes, 1944 y 1947; Vargas, 1947 y 1954; Ochoa, 2003, Cosio, 2005). El progenitor masculino "Yana Maqt'illo" fue elegido por su amplia adaptación, productividad y diferentes niveles de resistencia y tolerancia a plagas y enfermedades, así 
como por la calidad culinaria de sus tubérculos (Ver Figura 1 A)

SAYNO CONEJO Solanum tuberosum subsp. andigena (Juz. et Buk.) Hawkes $(2 \mathrm{n}=4 \mathrm{x}=48) \quad($ Hawkes, 1944 y 1947; Ochoa, 2003, Cosio, 2005). Este progenitor es de distribución muy restringida en las comunidades de Pacchanta y Lauramarca (3800 a 4200 Msnm) del Distrito de Ocongate -
Quispicanchis-Cusco. (Ver Figura 1 B). Se cultiva en terrenos muy cercanos al Nevado de Ausangate, con alta humedad relativa, que le ha permitido desarrollar resistencia a Phytophthora infestans y otras enfermedades. Planta de crecimiento alto, buena productividad $\mathrm{y}$ tiene tubérculos de carne amarilla de alta calidad culinaria.
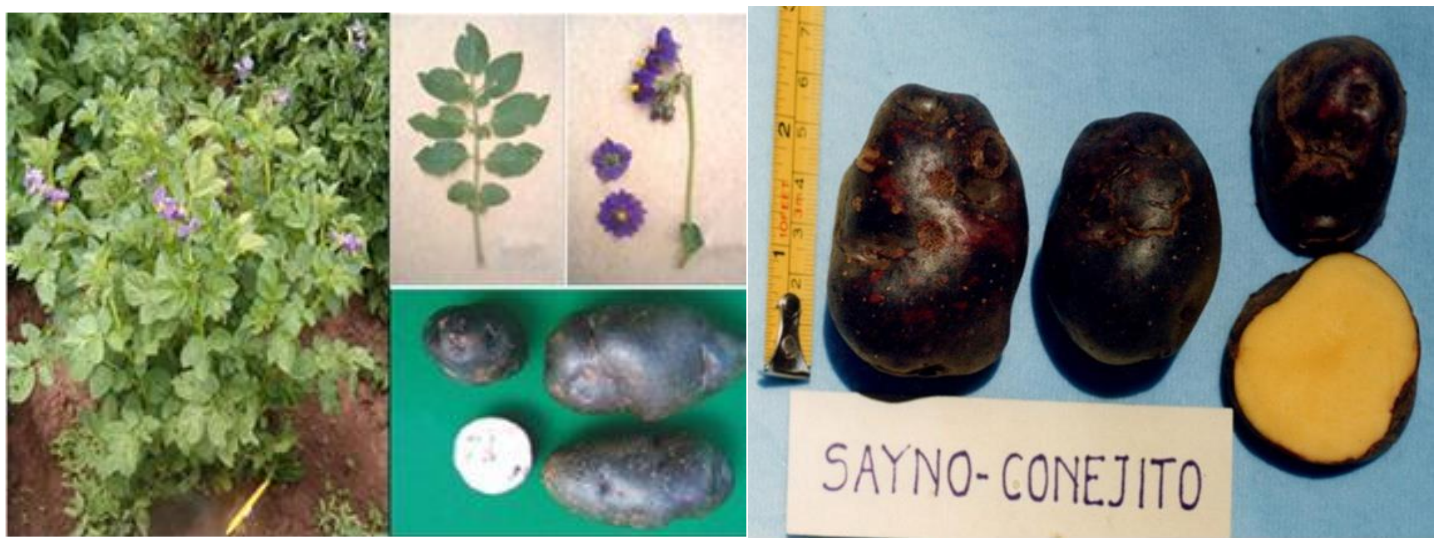

Figura 1. Izquierda. Papa nativa Yana Maqt illo progenitor masculino primera cruza; Derecha. Papa nativa Sayno Conejo progenitor masculino segunda cruza.

\section{Plan de cruzamientos y selección}

Se realizó una primera cruza utilizando como progenitor femenino Micaela Bastidas por "Yana Maqt'illo". (Estrada, 2000; Mendoza, 2008a y b). Las progenies fueron manejadas y evaluadas según los protocolos de mejoramiento genético en papa. De la cruza Micaela Bastidas/Yana Maqt'illo se obtuvieron 148 clones superiores que tuvieron la clave de KI (K'ayra I). Sobre esta población después de cuatro ciclos de evaluación y selección se identificó el Clon KI-43 como un genotipo promisorio, de rendimiento medio y relativa tolerancia a enfermedades, razón por la cual se procedió con la segunda cruza "KI-43" x "Sayno Conejo", en el año 2000. De esta cruza se obtuvieron 235 clones, que fueron evaluados a lo largo de nueve años, seleccionándose como el mejor al clon segregante "KISC-29B". Este clon pasó a la etapa de pruebas definitivas hasta su liberación como nueva variedad. La genealogía de la nueva variedad NUST'A-CICA, se muestra en la Figura 2. 
GENEALOGIADEL CULTIVAR ÑUST'A-CICA

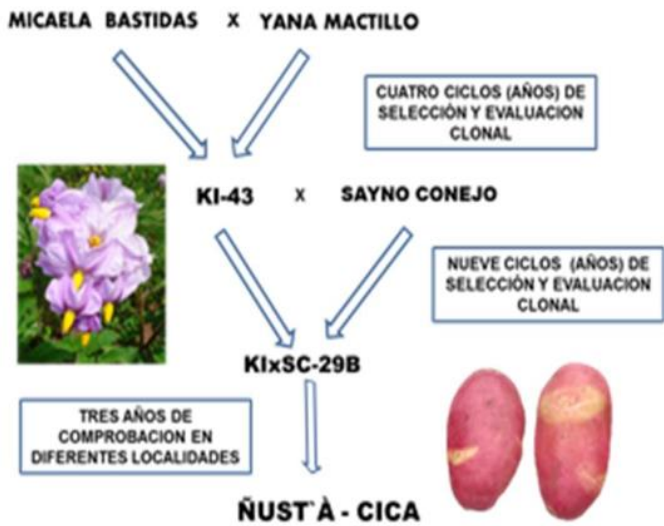

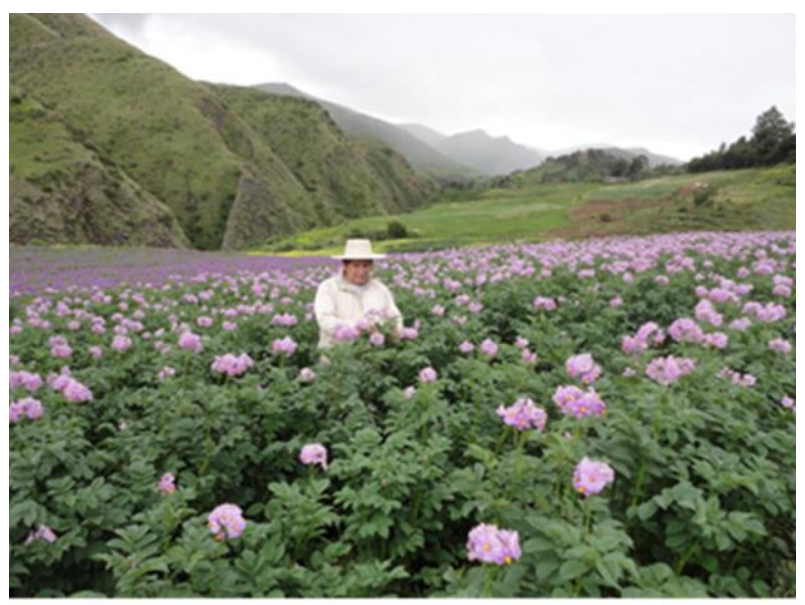

Figura 2. Izquierda. Genealogía del cultivar ÑUST'A-CICA; Derecha. El genetista comprueba la autoincompatibilidad de las flores en un semillero a $3800 \mathrm{msnm}$.

El clon "KI-43" tiene polen viable y estigmas receptivos, pero es autoimcompatible, esto quiere decir que "Yana Maqt'illo" tiene genes restauradores de androesterilidad, por cuanto Micaela Bastidas es androesteril con anteras muy deformadas. Se ha verificado que el $100 \%$ de plantas son autoincompatibles (Figura 2). Toda la progenie de esta cruza también es incompatible entre hermanos completos. El clon superior "KI-SC-29B" como progenitor masculino es compatible en diferentes cruzas con cultivares nativos tetraploides, así mismo, como progenitor femenino es totalmente viable en diferentes cruzas con cultivares nativos.

En doce ciclos de evaluación (doce años) hasta el 2012, con los híbridos en proceso de selección, se realizaron diez trabajos de tesis pre grado para ingeniero agrónomo sobre: rendimientos, resistencia a plagas y enfermedades y calidad culinaria del tubérculo. Durante este tiempo se seleccionó el clon "KI-43 x SC-29B", que finalmente se libera como la variedad "NUST'A-CICA".
La variedad NUST'A-CICA constituye progenie de las cruzas de Micaela Bastidas/Yana Maqt ${ }^{\prime}$ illo//Sayno Conejo, siendo un hibrido tetraploide autoincompatible, de la especie Solanum tuberosum spp. Andigena.

\section{Características básicas de la planta}

Habito de crecimiento erecto, con tallo pigmentado con abundante verde. Las alas del tallo son rectas. (Base teórica según Huamán 1986 y1994) (Figura 3 A).

La hoja es diseccionada, con el raquis moderadamente pigmentado en el haz y el envés totalmente verde. En promedio tiene cinco pares de foliolos laterales; dos pares de interhojuelas entre foliolos laterales; también tiene un par de interhojuelas sobre peciolulos. (figura 4). Los foliolos tienen coloración verde pálida con nervaduras amarillentas.

Todas estas caracteristicas indican que la variedad tiene un vigor muy superior a cualquier variedad nativa y aun a las comerciales como el testigo Canchan INIA, utilizado en los compartivos de rendimientos señalados en las diferentes tesis.

\section{Resultados y discusión}




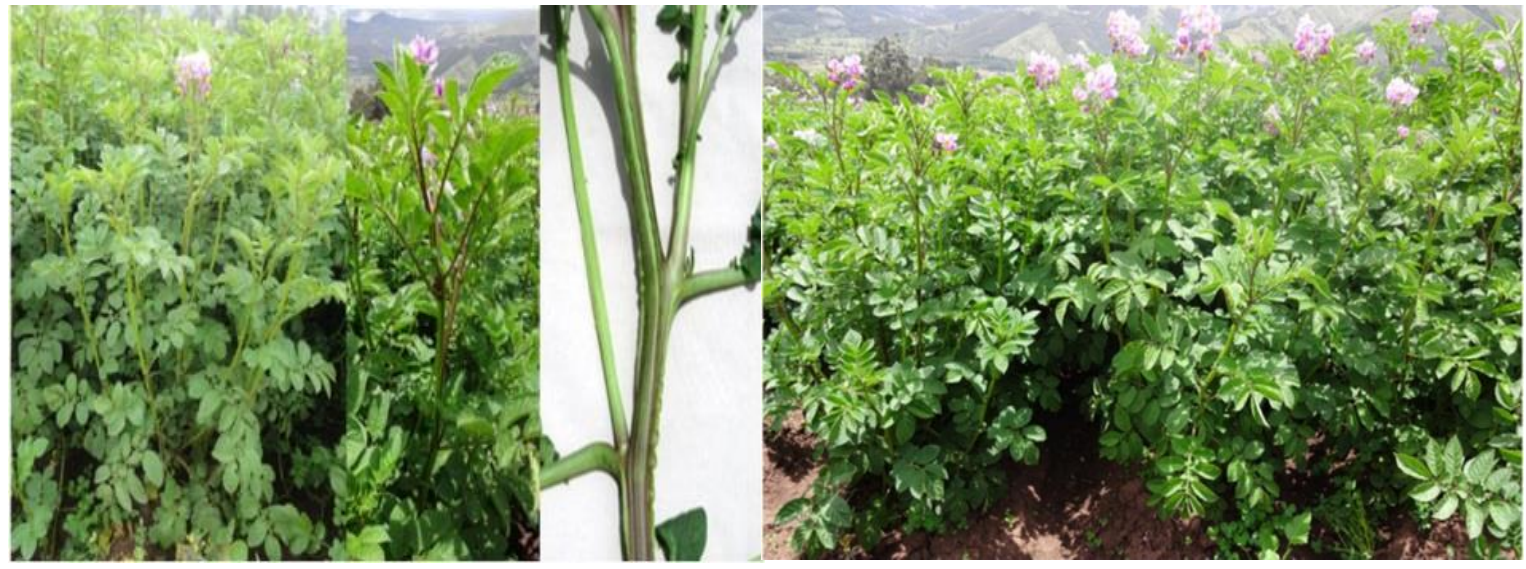

Figura 3.A: Características de planta y tallo. B: ÑUST'A-CICA en plena floración y libre de Phytophthora spp.

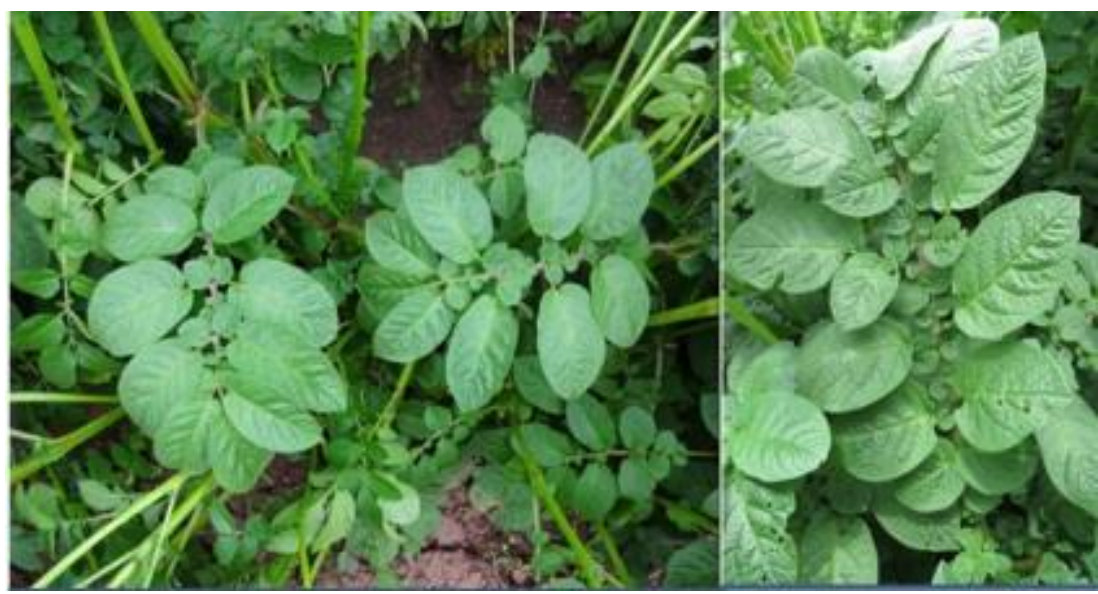

Figura 4: Hojas basales libre de daño de plagas y enfermedades.

Su grado de floración es moderada, el pedicelo pigmentado a lo largo y en la articulación. Cáliz pigmentado con abundante verde, de simetría regular. Corola de forma rotácea de color morado, de intensidad pálida, con color secundario de color violeta en la estrella. Presenta acumen blanco en el envés. Sin antocianina en anteras, estambres

normales.

Sin pigmentación en el pistilo. Forma de estigma capitado (Figura 5). La característica fundamental de esta variedad es que es auto incompatible, produce abundante polen viable $\mathrm{y}$ tiene estigma receptivo y no produce bayas. (Salas, 1993; Camadro, 2011; Maune, et al. 2010) 


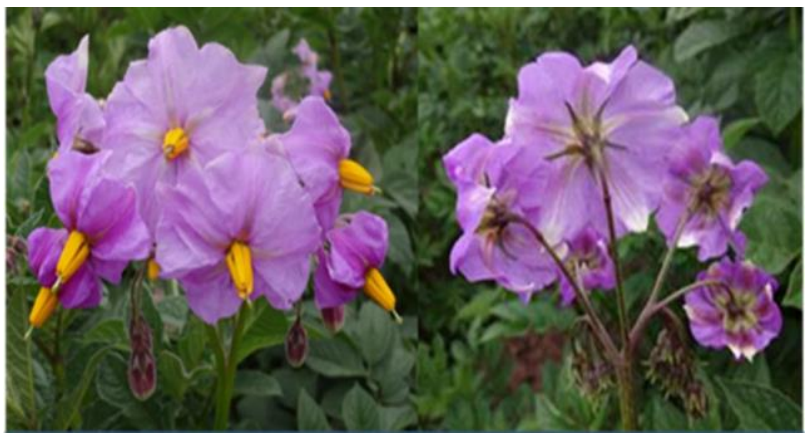

Figura 5: Inflorescencia con flores normales cuyas anteras producen abundante polen y estigmas bien desarrollados y receptivos.

El tubérculo es de color rojo-morado, de intensidad pálida, con color secundario amarillo distribuido en los ojos como anteojo (Figura 6). Color de carne amarillo, color secundario ausente; la forma del tubérculo es oblongo, con ojos superficiales, con piel lisa. Tiene brotes de color violeta sin color secundario.
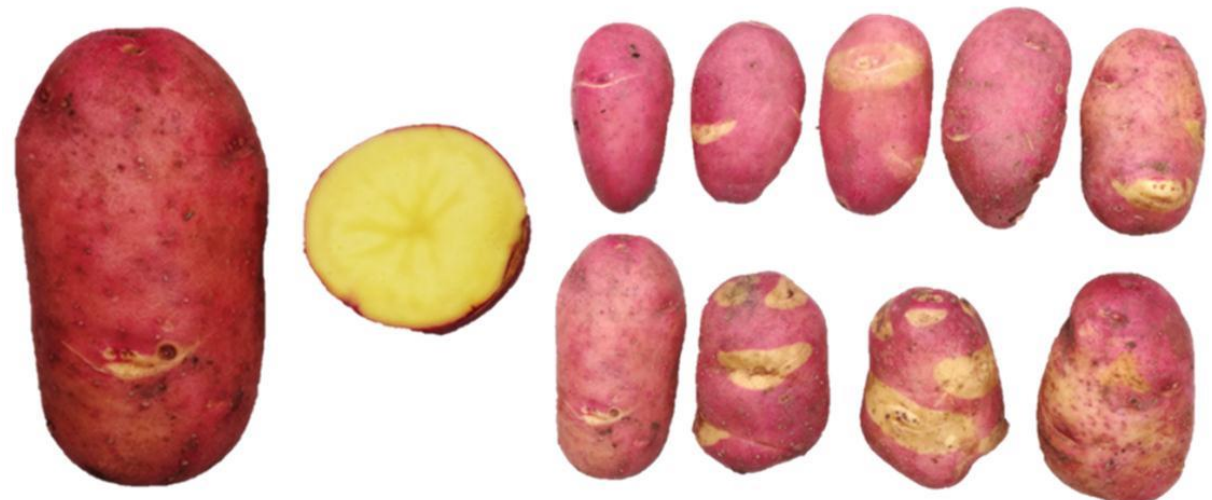

Figura 6: Tubérculos oblongos, rojo-morado, piel liza, carne amarilla.

Tiene brotes robustos muy ramificados y abundante raíz, presenta dominancia apical. El color del brote es morado intenso (Figura 7).

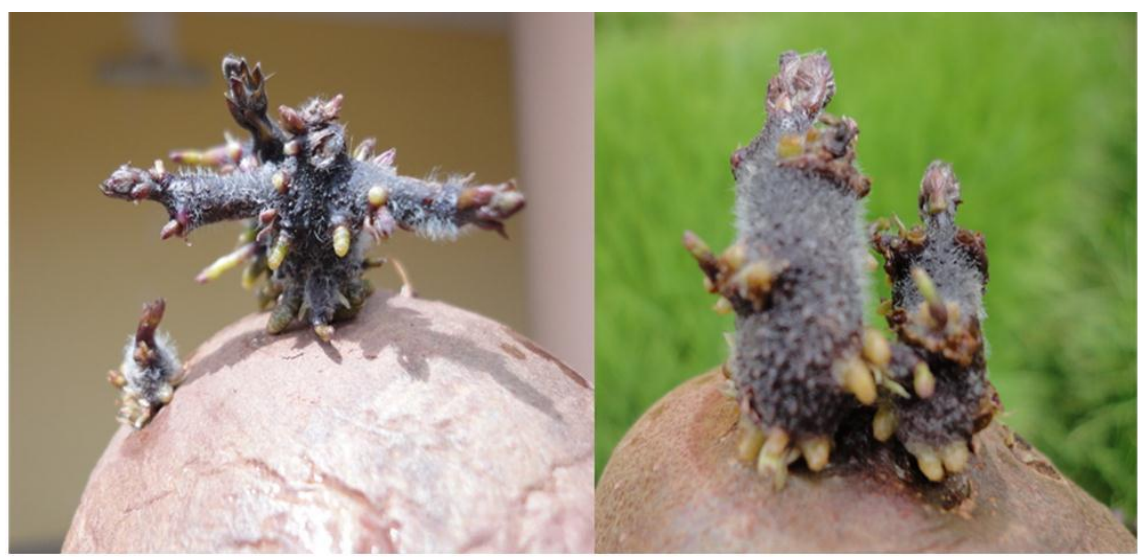

Figura 7:

Brotes color morado intenso, con abundante pubescencia, ramificación y raíces. 


\section{Evaluación de enfermedades y plagas}

La mayor bondad de esta nueva variedad es su alta resistencia a la rancha (Phytophthora infestans.), durante los 16 años de selección y continua a la fecha esta capacidad de resistencia. Esta variedad ha sido incluida en la prueba de resistencia de las variedades de Latinoamérica como parcela demostrativa en el $10^{\text {th }}$ World Congress Potato, instalado en la Estación Experimental Andenes INIA-Cusco, donde demostró su alta capacidad de resistencia.
En las diferentes tesis para ingeniero agrónomo, utilizando la escala de evaluación propuesta por el Centro Internacional de la Papa, (CIP, 1996; Barquero, 2005) demuestran fehacientemente la alta resistencia a Phytophthora, bajo diferentes condiciones ambientales de evaluación. se ha evaluado en diferentes lugares. En la Tabla 1 se exponen los resultados de evaluación del "tizón tardío"

Tabla 1. Grado de infección del "tizón tardío" a Ñust'a-CICA en diferentes localidades y años. Adaptado de (Pérez y Forbes, 2008) centro Internacional de la Papa.

\begin{tabular}{|c|c|c|c|c|}
\hline \multirow{2}{*}{$\begin{array}{c}\text { Evaluador Tesis: } \\
\text { Ingeniero } \\
\text { Agrónomo }\end{array}$} & \multirow[b]{2}{*}{ Lugar } & \multirow{2}{*}{$\begin{array}{l}\text { Altitud } \\
(\mathbf{m} . \text { s.n.m) }\end{array}$} & \multicolumn{2}{|c|}{$\begin{array}{l}\text { Grado de infección de } \\
\text { "Tizon Tardio" }\end{array}$} \\
\hline & & & $\begin{array}{l}\text { KIxSC- } \\
\text { 29B }\end{array}$ & $\begin{array}{l}\text { CANCHAN } \\
\text { (Testigo) }\end{array}$ \\
\hline \multirow{2}{*}{$\begin{array}{l}\text { Moscoso Donato } \\
\text { A. } \\
2002-2003\end{array}$} & Centro Agronómico Kayra. & 3240 & $\mathrm{G}=0$ & $\mathrm{G}=4$ \\
\hline & San Jerónimo - Cusco & & $\begin{array}{c}a \\
G=2\end{array}$ & $\begin{array}{c}\mathrm{a} \\
\mathrm{G}=6\end{array}$ \\
\hline \multirow{2}{*}{$\begin{array}{l}\text { Salcedo Rojas } \\
\text { Omar Froilan } \\
\text { 2009-2010 } \\
\end{array}$} & Chillimpampa - San Jerónimo & 3570 & $\mathrm{G}=0$ & $\mathrm{G}=5$ \\
\hline & - Cusco & & $\begin{array}{c}\mathrm{a} \\
\mathrm{G}=2\end{array}$ & $\begin{array}{c}\mathrm{a} \\
\mathrm{G}=7\end{array}$ \\
\hline \multirow{3}{*}{$\begin{array}{l}\text { Huarancca Levita. } \\
\text { Raúl } \\
\text { 2010-2011 }\end{array}$} & $\begin{array}{l}\text { Centro Agronómico Kayra. } \\
\text { San Gerónimo - Cusco }\end{array}$ & 3219 & \multirow{3}{*}{$\begin{array}{c}G=0 \\
a \\
G=2--\end{array}$} & \multirow{3}{*}{$\begin{array}{c}\mathrm{G}=3 \\
\mathrm{a} \\
\mathrm{G}=7\end{array}$} \\
\hline & Intipata. San Gerónimo-Cusco & 3370 & & \\
\hline & $\begin{array}{l}\text { Chillimpampa. San Gerónimo } \\
\text { - Cusco }\end{array}$ & 3570 & & \\
\hline \multirow{3}{*}{$\begin{array}{l}\text { Huisa Barrientos. } \\
\text { Roger. } \\
\text { 2010-2011 }\end{array}$} & $\begin{array}{l}\text { Huancco Pillpinto, Lamay- } \\
\text { Calca (parte alta) }\end{array}$ & 4070 & \multirow{3}{*}{$\begin{array}{l}G=0 \\
a \\
G=2\end{array}$} & \multirow{3}{*}{$\begin{array}{l}\mathrm{G}=3 \\
\mathrm{a} \\
\mathrm{G}=7\end{array}$} \\
\hline & $\begin{array}{l}\text { Huancco Pillpinto, Lamay - } \\
\text { Calca (parte media) }\end{array}$ & 3580 & & \\
\hline & $\begin{array}{l}\text { Huancco Pillpinto, Lamay - } \\
\text { Calca (parte baja) }\end{array}$ & 3377 & & \\
\hline $\begin{array}{l}\text { Cesar Augusto } \\
\text { Vara Quispe } \\
\text { (Agricultor) 2010- } \\
2011\end{array}$ & $\begin{array}{l}\text { Simataucca - Chinchero - } \\
\text { Urubamba }\end{array}$ & 2390 & $\begin{array}{l}G=0 \\
a \\
G=2\end{array}$ & $\begin{array}{c}G=3 \\
a \\
G=6\end{array}$ \\
\hline $\begin{array}{l}\text { Guillermo Ccoa } \\
2012-2013\end{array}$ & $\begin{array}{l}\text { Yutto- Andahuaylillas - } \\
\text { Quispicanchis }\end{array}$ & 3720 & $\begin{array}{c}\mathrm{G}=0 \\
\mathrm{a} \\
\mathrm{G}=2\end{array}$ & $\begin{array}{c}\mathrm{G}=3 \\
\mathrm{a} \\
\mathrm{G}=5\end{array}$ \\
\hline
\end{tabular}

En las evaluaciones en diferentes localidades y altitudes de la Región Cusco, se ha probado que Nust'a-CICA es resistente al daño de Phytophthora infestans, donde no se ha aplicado fungicida. Los bajos índices de daño permiten afirmar que la resistencia es de tipo horizontal, habiéndose registrado el 
daño en las hojas hasta un 3\% que corresponde al grado 2, que considera hasta más de 10 lesiones pequeñas por planta, detallando que la enfermedad solo prosperó a nivel de foliolos, pero no se observaron daños a nivel de tallo primario y secundario de la planta, tampoco en tubérculos. (Peña, 2009; Sanchez, 2011; Hilario, 2008)

Los insectos muestran poca preferencia de consumo de esta variedad, el Epitrix spp y la Diabrotica spp, al inicio del desarrollo de las plantas ocasionan daños moderados en los foliolos, posteriormente las plantas por su precocidad y su alto vigor de desarrollo, generan el escape o alta tolerancia a los masticadores de hojas. (Cosio, et al. 2012; Salcedo 2013).

Los daños ocasionados por los barrenadores del tallo (Atomopteryx sp y Synmestrichema tangolias), constituidas como plaga clave a nivel de campo, y esqueletizador de las hojas (Tequus sp), han registrado daños de escasa a moderada. Por otro lado, los barrenadores de los tubérculos: Premnotrypes spp en campo, Pthorimaea opercullela $y$ Synmestrichema tangolias (polilla de la papa) en almacén, no han mostrado daños importantes, (Misme, 2003; Quispe, 2014). Las larvas del primer y segundo estadio de Premnotrypes spp llegan a ocasionar daños superficiales en los tubérculos, porque, se observa un efecto de antibiosis de un pigmento rojo que genera una capa corchosa como barrera y evita la continuidad de daño de las larvas. (Salcedo, 2013)

\section{Calidad culinaria}

Con los trabajos de tesis, se ha realizado las evaluaciones de calidad culinaria, donde se evaluó las variables que se muestran en Tabla 2. (Moscoso, 2004 y Quispe, 2007).

Tabla 2. Evaluaciones de materia seca, calidad culinaria en sancochado y fritura.

\begin{tabular}{ll}
\hline Nust'a CICA (Clon KI x SC-29B) & Características \\
\hline Porcentaje de materia seca & $25.78 \%$ (Media) \\
Color de pulpa en sancochado & Amarillo \\
Sabor en sancochado & Agradable \\
Textura en sancochado & Harinoso \\
Facilidad de pelado en sancochado & Muy fácil \\
Tiempo de cocción (sancochado) & 10 minutos iniciado el hervor \\
Color después de la fritura & Amarillo \\
Consistencia después de la fritura & Suave \\
Sabor en la fritura & Muy agradable \\
Absorción de aceite & Medio \\
Oxidación en fritura & Ligera \\
Oxidación a 24 horas después & Ligera \\
Tiempo de cocción en fritura & 04 minutos \\
Numero de tubérculos/planta & 16 promedio \\
\hline
\end{tabular}

Esta variedad tiene materia seca superior a $26 \%$, que le confiere cualidades culinarias deseables para su consumo como en frituras por su baja oxidación, elaboración de pastas o purés en sopas y sancochados. 


\section{Ensayos de adaptación}

Los ensayos de adaptación a partir del año 2011 al 2013, fueron realizados en ambientes que variaron de 2930 a 4070 Msnm (Wissar, 1988, Gabriel, 2010). El genotipo (KI-SC-29B) NUST ${ }^{\prime} A-C I C A$ en altitudes menores a 3000 Msnm, mostró un rendimiento promedio de 24 $\mathrm{t} / \mathrm{ha}^{-1}$. Los mejores rendimientos se dieron entre 3200 a 4070 Msnm siendo en promedio $40 \mathrm{t} / \mathrm{ha}^{-1}$ lo que muestra que el genotipo está recomendado para altitudes mayores a 3200 Msnm, como son los ámbitos de la sierra sur del país, preferentemente en los departamentos de Cusco, Apurímac y Puno u otras zonas andinas con rango de variación altitudinal similares.

\section{Conclusiones}

La variedad NUST'A-CICA es una especie tetraploide, con amplia adaptación a diferentes ambientes altitudinales, desde 3200 a mayor de 4000 Msnm, que le otorga al agricultor mayores posibilidades de producción con rendimientos superiores a $40 \mathrm{t} / \mathrm{ha}^{-1}$.

Esta variedad se ha comportado de manera estable con alta resistencia probada a Phytophthora infestans en diferentes ambientes y épocas de siembra. También muestra la poca preferencia al daño de los insectos masticadores de hojas y barrenadores de tubérculos, plagas propias de la zona andina.

Esta variedad constituye el primer reporte de papas tetraploides autoincompatibles, se ha demostrado que es autoincompatible, por cuanto no produce bayas y probado mediante cruzas con otras variedades tetraploides la viabilidad del polen y la receptividad de los estigmas, que será objeto de un reporte posterior.

\section{Agradecimientos}

Los autores agradecen al Centro de Investigación en Cultivos Andinos CICAFCA-UNSAAC por las facilidades brindadas para la realización del presente trabajo de investigación. También se agradece a la UNSAAC, por el financiamiento del Proyecto: "CONSERVACION EX SITU DE TARWI, MAIZ, PAPA NATIVA Y LA LIBERACION DE VARIEDADES PARA LA AGRICULTURA REGIONAL" financiado con fondos de CANON.

\section{Conflictos de intereses}

Los autores declaran que la presente publicación no presenta conflictos de interés con ninguna persona o institución.

\section{Referencias citadas}

Barquero, M.; Gómez, L.; Brenes, A. (2005). Resistencia al tizón tardío (Phytophthora infestans) en clones promisorios de papa en costa rica1/. Agronomía Costarricense 29(3): 31-45. Recuperado de https:www.mag.go.cr/rev agr/inicio.htm www.cia.ucr.ac.cr

Camadro, E. L. (2011). Hibridación y flujo génico en especies silvestres de papa de la Argentina. Journal Basic Applied Genetics 22(1): 2.

Centro Internacional De La Papa (CIP). (1996). Principales Enfermedades, Nematodos e Insectos de la Papa. CIP, Lima, Perú.

Cosio C. P.; Catalán B. W.; Yépez Ch. E. (2012). Sistematización de Información sobre la caracterización y evaluación de híbridos de papa durante 15 ciclos de Selección. Cusco, Perú.

Cosio, C. P. (2005). Conocimiento de la Variabilidad de Papas en las 
Comunidades Conservacionistas del Cusco. Proyecto In Situ - Cusco - Perú.

Estrada R.N. (2000). La biodiversidad en el mejoramiento genético de papa. CIPIPGRI. La Paz, Bolivia.

Gabriel, J. (2010). Estrategias y perspectivas del mejoramiento genético de papa (Solanum tuberosum L.). Cochabamba, Bolivia. Recuperado de https://www.proinpa.org/tic/pdf/Papa/Var ios\%20Papa/Estrategias\%20y\%20perspec tivas $\% 20$ del $\% 20$ mejoramiento $\% 20$ geneti co\%20de\%20papa\%20en\%20Bolivia.pdf

Hawkes J. G. (1944). Potato Collecting Expeditions in Mexico and South America. II. Systematic classification of the Collections. Imperial Bureau of Plant Breeding and Genetics. School of agriculture. Cambridge. Inglaterra.

Hawkes J.G. (1947). On the origin and meaning of South America Indian potato names. Journal Linn. Soc. Botany. 53(50): $205-250$.

Hilario C, R. (2008). Comparativo de Rendimiento de Seis Clones Promisorios de papa (Solanum andigenum spp andigenum) Bajo Condiciones del Centro Agronómico Kayra. Tesis grado Universidad Nacional de San Antonio Abad del Cusco. Facultad de Agronomía y Zootecnia Cusco, Perú.

Huamán, Z. (1986). Botánica sistemática y morfología de la Papa. Boletín de Información Técnica 6. Centro Internacional de la Papa. Lima, Perú. 22pp.

Huamán, Z. (1994). Descriptores de papa, Centro Internacional de la Papa CIP Lima - Perú

Huisa B, R. (2013). Comparativo de Rendimiento de Cinco Clones de Papa (andigena $\mathrm{x}$ andigena) y un Testigo en Tres Localidades de la Comunidad de
Huancco Pilpinto, Lamay - Calca. Tesis grado Universidad Nacional de San Antonio Abad del Cusco. Facultad de Agronomía y Zootecnia Cusco, Perú.

Maune J.F.; Camadro E.L.; Pontaroli A.C. (2010). Incompatibilidad cruzada y autoincompatibilidad gametofítica en familias e híbridos interespecíficos de papa. XIV Congreso Latinoamericano de Genética (ALAG). Viña del Mar, Chile.

Mendoza, H. H. (2008). Genética cuantitativa y mejoramiento de papas autotetraploides. CIP. Lima, Perú.

Mendoza, H. H. (2008). Genética y mejoramiento de la papa en el Perú. CIP. Lima, Perú.

Misme M, M A. (2003). Selección de 156 Híbridos de Tres Cruzas de Papa por Rendimiento en Condiciones de Kayra. Tesis grado Universidad Nacional de San Antonio Abad del Cusco. Facultad de Agronomía y Zootecnia Cusco, Perú.

Moscoso A, D. (2004). Selección de Híbridos de Papa por Rendimiento de Tubérculo, Materia Seca y Calidad Culinaria Bajo Condiciones del Centro Agronómico Kayra. Tesis grado Universidad Nacional de San Antonio Abad del Cusco. Facultad de Agronomía y Zootecnia. Cusco, Perú.

Ochoa, C. (1990). The potatoes of South America. Bolivia. Cambridge University Press. Cambridge, Inglaterra.

Ochoa, N.C. (2003). Las papas del Perú.Base de datos 1947 - 1997. CIP, UNALM, COSUDE. Lima, Perú.

Peña Ch, G. (2009). Comparativo de Rendimiento de Seis Híbridos de Papa (Solanum andigenum spp andigenum). Tesis grado Universidad Nacional de San Antonio Abad del Cusco. Facultad de Agronomía y Zootecnia Cusco, Perú. 
Pérez, W.; Fobres, G. (2008). El tizón Tardío de la Papa - Centro Internacional de la papa (CIP). Lima, Perú.

Quispe G, I. (2007). Selección de Híbridos de Papa por Materia Seca y Calidad de Tubérculo". Tesis grado Universidad Nacional de San Antonio Abad del Cusco. Facultad de Agronomía y Zootecnia. Cusco, Perú.

Quispe H, J. (2014). Comparativo de Rendimientos de Seis Híbridos de Papa (andigena $\mathrm{x}$ andigena) en Siembra Temprana Bajo Condiciones del Centro Agronómico Kayra-Cusco. Tesis grado Universidad Nacional de San Antonio Abad del Cusco. Facultad de Agronomía y Zootecnia. Cusco, Perú.

Salas, A. (1993). Incompatibilidad cruzada entre cinco especies tuberosas de Solanum (Solanácea). Darwiniana 92(1:4)18.25.
Salcedo R, O. (2013). Daño de Plagas y Enfermedades en 27 Híbridos de Papa Bajo Condiciones de Centro Agronómico Kayra. Tesis grado Universidad Nacional de San Antonio Abad del Cusco. Facultad de Agronomía y Zootecnia. Cusco, Perú.

Sánchez S, G. (2011). Comparativo de Rendimiento y Respuesta a Plagas y Enfermedades de 09 Híbridos de Papa Bajo Condiciones de Centro Agronómico Kayra. Tesis grado Universidad Nacional de San Antonio Abad del Cusco. Facultad de Agronomía y Zootecnia. Cusco, Perú.

Vargas C. C. (1947). Las papas SubPeruanas. Parte I. Publicaciones de la Universidad del Cusco. Cusco, Perú.

Vargas C. C. (1954). Las papas SubPeruanas. Parte II. Publicaciones de la Universidad del Cusco. Cusco, Perú.

Wissar, R. Ruiz, R. (1988). Mejoramiento de la papa en el CIP por adaptación a climas tropicales. Lima-Perú. 